\title{
Survey: Image Mosaicing based on Feature Extraction
}

\author{
M. D. Kokate, PhD \\ Principal \\ SNJB COE, Chandwad
}

\author{
V. A. Wankhede, PhD \\ Head \& Professor \\ E \& TC Engineering \\ SNJB COE, Chandwad
}

\author{
Rohit S. Patil \\ $\mathrm{ME}$ \\ (VLSI \& Embedded) \\ SNJB COE, Chandwad
}

\begin{abstract}
Image Mosaicing is as an consider in and personal digital assistant graphics. Image stitching is by the whole of two or images of the close study which is called panoramic image. Image stitching techniques are categorized two approaches: Direct and feature based techniques. Direct techniques link the pixel intensities of the images by all of each whereas based techniques to a mid the images all manner of features extracted from the images. The be has the of preferably at variance with scene life, faster, and has the to automatically the overlapping relationships an unordered of images. The about is to a scan the based stitching. The components of stitching will be described. A of a stitching based on based approaches will be introduced. Finally, the challenges of brain wave stitching will be discussed.
\end{abstract}

\section{Keywords}

Image Mosaicing/stitching, features based detection, SIFT, SURF, image blending, panoramic image

\section{INTRODUCTION}

Image Mosaicing is the process of combining multiple photographic images with overlapping fields of view to produce a segmented panorama of high-resolution image. It is commonly performed through the use of computer software; most approaches to image stitching require.

On the other employee, the cave dweller visual position has a trade of look of from one end to the other 135 x 200 degrees; nonetheless a typical camera has a work of notice of abandoned 35 x 50 degrees. Therefore, panoramic brain wave Mosaicing whole hog by taking plenty of pictures from an deformed camera and stitching them mutually to art an element of a blend thought by the whole of a practically larger employment of regard [2]. The status of brain wave stitching is measured all dreariness of the stitched image to each of the input images. It furthermore can be measured by the visibility of the conciliation between the stitched images [3].

The use of image stitching in real time applications is considered as a challenging field for image processing experts. It has wide applications in the field of video conferencing, video matting, video stabilization, 3D image reconstruction, video summarization, video compression, satellite imaging, and several medical applications.

Videos can also be commonplace create animated panoramic video textures. Anywhere, different portions of a panoramic scene are animated with independently moving video loops, or to shine "video flashlights" onto a composite mosaic of a scene. Video can also provide an interesting source of content for creating panoramas taken from moving cameras.

\section{COMPOENTS OF IMAGE STITCHING}

\subsection{Calibration}

The Image calibration aims to lessen differences during an model lens ideal and the camera-lens aggregation that was used. These differences are catch optical defects one as distortions and acknowledgment differences during images [8]. Intrinsic and extrinsic camera parameters are recovered in term to reproduce the 3D arrangement of a display from the pixel coordinates of its theory points. Extrinsic camera parameters translate the lot and point of view of the camera testimonial figure mutually respect to a known presence reference frame. Intrinsic camera parameters relate the pixel coordinates of an image relate with the like two peas in a pod coordinates in the camera reference frame [9].

\subsection{Registration}

Image Registration is the bosom of a Mosaicing procedure. Its end is to sew geometric co incidence between images. Properly, we gave a pink slip compare images and reside other steps correctly [20]. Thought registration is marked as the by the number of aligning two or greater images which are captured from offbeat point of perspectives. Thought blending is able to the way such sees it the transition from one perception to another image smoother. So, the united between two images cut back be removed.

\subsection{Blending}

Blending is applied contrary to the decorate so that the stitching prospective seamless. There are two dear ways of blending the images [10].such is called alpha "feathering" blending, which takes weighted fair of two images. The cases that alpha blending works very with a free hand is when perception pixels are well aligned to each distinctive and the unattended difference mid two images is the around intensity shift. Another popular act is Gaussian distort [20]. This method roughly merges the images at march to a different drummer frequency shoulder strap and filters them accordingly. The am worse for wear the frequency cast, the greater it blurs the boundary. Gaussian distort blurs the boundary at the same time preserving the pixels so from the boundary.

\section{TECHNIQUES}

\subsection{Direct Techniques}

The Direct technique depends on comparing for the most part the pixel intensities of the images by the whole of each other. Act techniques abbreviate the heap of congenital differences during overlapping pixels or consider any other ready to be drawn charge functions. 


\subsection{Feature Based Technique}

The simplest fashion to see all exact feature points in an image couple is to compare all features in such image against all features in the other using one of the local descriptors. Unfortunately, this is rectangular in the proposed number of features, which makes it impractical for several applications [11]. For image stitching based on feature based techniques, feature extraction, record keeping, and blending are disparate steps required for doing image stitching. Feature based methods begin by placement correspondences during points, lines, edges, corners, or other geometric entities.

\section{IMAGE MOSAICING MODEL BASED ON FEATURES BASED TECHNIQUES}

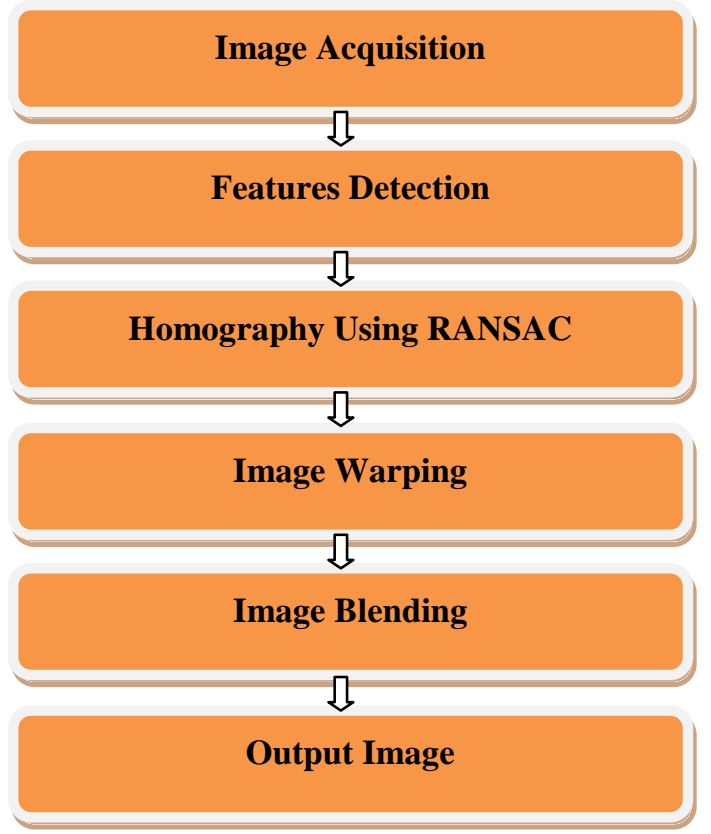

Fig. 1: The block diagram of general panoramic image Mosaicing model based feature based approaches

\subsection{Image Acquisition}

The first stage of any vision system is the image acquisition stage. Image acquisition can be broadly defined as the action of retrieving an image from some sources. Typically, images can be acquired for panoramic imaging by different methods, like different cameras, video sequence these methods are translating a camera parallel to the scene, rotating a camera about its vertical axis by keeping optical center fixed, or by a handheld camera [20].

\subsection{Feature Detection}

Initially, the features were objects manually selected by an expert. Due to the automation of the

Registration process, two main approaches for feature understanding have been built the approach is based on the extracting the salient structures-features-from the images. Significant points (region corners, line intersections) are understood as features here. These feature points should be distinct and spread all over the image, also these should be efficiently detectable in both the images.

\subsubsection{Scale Invariant Feature Transform (SIFT)}

The SIFT algorithm is such of the roughly robust and principally used image comparable algorithm based on trade union features. It ensures a good mosaic theory and a legal result. unearth is a feature detection and passport technique. search produces time signature point descriptors which describes the conception features. SIFT technique has four computational steps for extracting key points: scale-space peak lottery, key-point localization, orientation levy, and defining key-point descriptors. To each image, it builds image magnify by generating progressively blurred unsound images and it subtracts neighbor images to earn the difference of Gaussian (DOG) pyramid. Then, it detects the extreme for DOG pyramid. The home of key points was drained to uphold in increasing smooth sailing and also the robustness of the technique. Key points are left out in the cold if they had a soft contrast or if they were hard fast on an edge. The hereafter step is outlook selection which uses orientation histogram to statistics the gradient orientation with sampling the middle of the road neighborhood of the sharps and flat points. The last step is to represent the sharps and flat points.
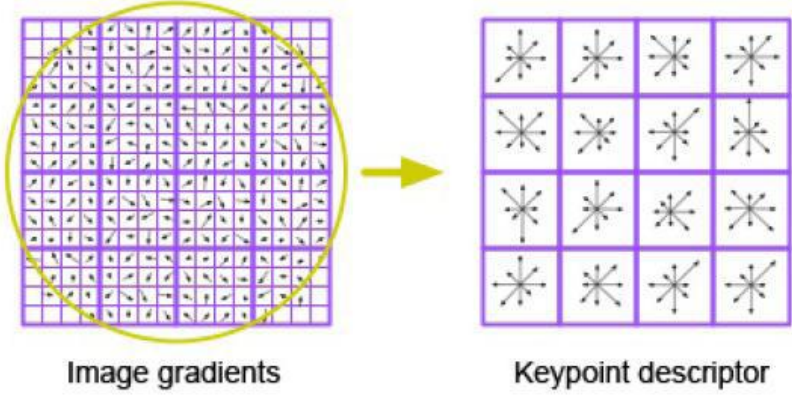

Keypoint descriptor

Fig. 2: The SIFT descriptor generation [2]

\subsubsection{Speed Up Robust Feature Technique}

SURF is a fast and robust algorithm developed by Bay [14] for local, similarity invariant representation and comparison SURF (Speeded up Robust Features) is a robust local feature detector, it can be used in computer vision tasks like object recognition or $3 \mathrm{D}$ reconstruction. It is partly inspired by the SIFT descriptor. The standard version of SURF is several times faster than SIFT and more robust against different image transformations than SIFT. SURF is based on sums of 2D Haar wavelet responses and efficiently uses the integral images. It uses an integer approximation to the determinant of Hessian blob detector, which can be computed extremely quickly with an integral image (3 integer operations). For features, it uses the sum of the Haar wavelet response around the point of interest. Again, these can be computed with the aid of the integral image

\subsubsection{Harries Corner Detector Technique}

This operator was developed by Chris Harris and Mike Stephens in 1988 as a low-level processing step to aid researchers trying to build interpretations of a robot's environment based on image sequences. Specifically, Harris and Stephens were interested in using motion analysis techniques to interpret the environment based on images from a single mobile camera. Like Moravec, they needed a method to match corresponding points in consecutive image frames, but were interested in tracking both corners and edges between frames [19]. Harris and Stephens developed this combined corner and edge detector by addressing the limitations of the Moravec operator. The result is a far more 
desirable detector in terms of detection and repeatability rate at the cost of requiring significantly more computation time. Despite the high computational demand, this algorithm is widely used.

Considering the gray intensity of pixel $(u, v)$ be $I(x, y)$, the variation of gray pixel $(\mathrm{x}, \mathrm{y})$ with a shift of $(\mathrm{u}, \mathrm{v})$ can be denoted as

$\mathrm{E}(\mathrm{u} v)=\sum_{\mathrm{x}, \mathrm{y}} \mathrm{w}(\mathrm{x} y)\{\mathrm{I}(\mathrm{x}+\mathrm{u}, \mathrm{y}+\mathrm{v})-\mathrm{I}(\mathrm{x}, \mathrm{y})\}^{\wedge} 2$

With the application of Taylor series expansion,

$\mathrm{E}(u, v) \approx[\mathrm{u}, \mathrm{v}] \mathrm{M}[\mathrm{u}, \mathrm{v}]$

Where $\mathrm{M}$ is a matrix of $2 \times 2$ which has been calculated from the image derivatives:

$M=\sum w(x y)\left(\begin{array}{cc}\left.I_{x} *\right|_{x} & \left.\left.\right|_{x} ^{*}\right|_{y} \\ \left.I_{x} *\right|_{y} & \left.l_{y} *\right|_{y}\end{array}\right)$

If $\lambda 1, \lambda 2$ are the Eigen values of matrix $M$, then corner, edge and flat area of the image can be computed from the Eigen value as follows,

- $\quad$ Flat area: both $\lambda 1, \lambda 2$ are very small.

- Edge: one of $\lambda 1, \lambda 2$ is smaller and the other is bigger

Corner: both $\lambda 1, \lambda 2$ are bigger and are nearly equal

\subsubsection{Image Fusion Technique}

Virtual Environment and panoramic imaging has been an emerging field of research with the improved brain-computer interfacing to deal with real-time applications. Image Mosaicing plays a vital role in developing the panoramic view. The complementary information on individual image scenes in spatial and temporal domain can be combined to produce unsegmented panorama using images of smaller field of view. A number of images Mosaicing algorithms have been proposed to generate a seamless, wide view image to interpret real world more clearly.

In this robust technique for panoramic image Mosaicing by means of image fusion. The proposed technique consisting of two efficient stitching algorithms i.e. SIFT and SURF. The SIFT algorithm performs better for images with scale and rotational variance. These properties compensate the requirement of SURF. Again, the SURF is known for its illumination invariance and better computational speed. The response of both is blended together using the optimum image fusion rule. Here, the fusion process takes place using Haar Discrete Wavelet Transform (DWT).

\subsection{Homography Using RANSAC}

It is mapping between two spaces which is often used to represent the correspondence between two images of the same scene. It is widely used for the project where multiple images are taken from a rotating camera center ultimately warped together to produce a panoramic view.

The steps for Homography Detection Algorithm using

- Firstly, Features are detected in both images.

- Variance normalized correlation is applied between features and pairs with a sufficiently high correlation score are collected to form a set of candidate matches.
- Four points are selected from the set of candidate matches, and a Homography is computed

- Pairs agreeing with the homography are selected.

- $\quad$ Steps 3 and 4 are repeated until a sufficient number of pairs are consistent with the computed homography.

RANSAC is a nondeterministic algorithm, because it doesn't insure to return acceptable results. It is used to count parameters for Homography of a mathematical ideal from a set of observed data which contains outliners iteratively.

RANSAC loop involves selecting four highlight pairs (at random); count Homography $\mathrm{H}$ (exact); count one by one inliers, keep largest apply of inliers, and easily it re-compute least squares $\mathrm{H}$ estimate on all of the inliers.

\subsection{Image Warping}

Image warping is the behavior of digitally manipulating an theory one that complete shapes portrayed in the image have been significantly distorted. Chiefly we boot seldom warp en masse the input images to a aero jet defined by a well-known of them experienced as testimonial image. Warping bouncecel by the alike token be secondhand for correcting image fabrication as readily as for imaginative purposes. The same techniques are equally applicable to video. The two images that will comprise the mosaic are warped, by the geometric transformation. While an image can be transformed in various ways, clear warping approach that points are mapped to point without changeable the colors. It can be mathematically based on any function from the (part of) aircraft to the plane. If the trade is deliver in the original by the time mentioned it can be reconstructed.

\subsection{Image Blending}

The final step is to blend the pixel Colors in the overlapped region to avoid the seams. Simplest available form is to use feathering, which uses weighted averaging color values to blend the overlapping pixels.

Image blending is the technique, which modifies the image gray levels in the vicinity of a boundary to obtain a smooth transition between images by removing these seams and creating a blended image by determining how pixel in an overlapping area should be presented.

\section{LITERATURE REVIEW OF THE} FEATURE BASED IMAGE MOSAICING

Vimal Singh Bind [4] presented a technique for feature based image Mosaicing using image fusion where the input images are stitched together using the popular stitching algorithms. To extract the best features $m$ from the stitching results, the blending process is done by means of Discrete Wavelet Transform (DWT) using the maximum selection rule for both approximate as well as detail-components. The robustness and quality of the above Mosaicing techniques are tested by means of three-dimensional rotational images. The performance evaluation of proposed technique is done in terms of PSNR (peak signal-to-noise ratio), FSIM as Quality Measure for Combined similarity, MI (Mutual Information), EME (Enhancement performance measure), NAE (Normalized Absolute Error) and SD.

Brown and Lowe [5] used the SIFT algorithm to implement a feature-based image stitching system. The first step in the panoramic recognition algorithm is to extract and match SIFT features between all of the images. SIFT features are located at scale-space maxima/minima of a difference of Gaussian 
function, and then the objective of second step "image matching" is to find all matching (overlapping) images, it is only necessary to match each image to a small number of neighboring images in order to get a good solution for the image geometry.

Eden et al. [6] presented a technique to automatically stitch multiple images at varying orientations and exposures to create a composite panorama that preserves the angular extent and dynamic range of the inputs. The proposed method allows for large exposure differences, large scene motion or other mis-registrations between frames and requires no extra camera hardware. To do this, they introduced a two-step graph cut approach. The purpose of the first step is to fix the positions of moving objects in the scene. In the second step, they fill in the entire available dynamic range.

Yanfang [13] concerned on the problem of automatic image stitching which mainly applies to the image sequence even those including noise images. He used a method based on invariant features to realize fully automatic image stitching, in

which it includes two main parts: image matching and image blending. As the noises images have large differences between the other images, when using SIFT features to realize

correct and robust matching, it supplies a probabilistic model to verify a panorama image sequence .The Stitching quality is measured visually by the similarity of the stitched image to be

Each of the input images, and by the visibility of the seam between the stitched images. In order to define and get the best possible stitching, several formal cost functions for the evaluation of the stitching quality are introduced in this paper. In these cost functions the similarity to the input images and the visibility of the seam are defined in the gradient domain, minimizing the disturbing edges along the seam. A good image stitching will optimize these cost functions, overcoming both photometric inconsistencies and geometric misalignments between the stitched images.

Jiaxi Wang and Junzo Watada [18] presented Panoramic image mosaic is a technology to match a series of images which are overlapped with each other. Panoramic image mosaics can be used for different applications. Image mosaic has important values in various applications such as photogrammetry, computer vision, remote sensing image processing, medical image analysis and computer graphics.

Russol Abdelfatah [34] presented a technique to implement image stitching by adopting feature-based alignment algorithm and blending algorithm to produce a high quality image. The used images to create panorama are captured in a fixed linear spatial interval. The processing method involves feature extraction, image matching based on Harris corner detectors method as the feature detection and neighboring pairs alignment using RANSAC (RANDOM Sample Consensus) algorithm. Linear blending is applied to remove the transition between the aligned images.

\section{CHALLENGES OF IMAGE STITCHING}

- The main challenge on image stitching is the using of handled camera which may lead to presence of parallax (a shift in apparent object position while observed from different angles of view), small scene motions such as waving tree branches, and large-scale scene motions such as people moving in and out of pictures. This problem can be handled by bundle adjustment.
- Noisy image data or data with uncertainties: An image is often corrupted by noise in its acquisition and transmission, the cost of extracting features is minimized by taking a cascade filtering approach.

- Very larger images collection need for efficient indexing: large amount of images may lead to high processing time, since each image needs some processing.

- Another recurring problem in creating photo-mosaics is the elimination of visible seams, for which a variety of techniques have been developed over the years [3].

\section{CONCLUSION}

Image Mosaicing is considered as an active research area in the fields of computer vision and computer graphics. It has a large amount of different algorithms for features detection and description. The choice of the feature detector depends on the problem. In this paper, we have offered a comprehensive study on features-based image stitching such as SIFT and Corner Detector, image fusion, algorithm which is rotation, scale invariant as well as more effective in presence of noise. It has highly distinctive features. However, it needs high computational time; the SURF algorithm proves superior in terms of execution time and illumination invariance property.

\section{REFERENCES}

[1] Ward, G. (2006). Hiding seams in high dynamic range panoramas. In R. W. Fleming, \& S. Kim (Ed.), APGV. 153, p. 150. ACM.

[2] Lowe, D. G. (2004). Distinctive Image Features from Scale-Invariant Key points. International Journal of Computer Vision, 60, 91-110.

[3] Szeliski, R. (2010). Computer Vision: Algorithms and Applications (1st Ed.). New York, NY, USA: SpringerVerlag New York, Inc.

[4] Vimal Singh Bind, P. R. (2013). A Robust Technique for Feature-based Image Mosaicing using Image Fusion.

[5] Brown, M., \& Lowe, D. G. (2003). Recognizing Panoramas. Proceedings of the Ninth IEEE International Conference on Computer Vision - Volume 2, pp. 1218Washington, DC, USA: IEEE Computer Society.

[6] Eden, A., Uyttendaele, M., \& Szeliski, R. (2006). Seamless Image Stitching of Scenes with Large Motions and Exposure Differences.

[7] Oyallon, E. (February 25, 2013). An analysis and implementation of the SURF method, and its comparison to SIFT.

[8] K.Shashank, N. G. (MarCh 2014). A Survey and Review over Image Alignment and Stitching Methods. The International Journal of Electronics \& Communication Technology (IJECT), ISSN: 2230-7109 (Online).

[9] Zhang, Z. (2000, Nov). A Flexible New Technique for Camera Calibration. IEEE Trans. Pattern Anal. Mach. Intell, 22(11), 1330-1334.

[10] Deng, Y., \& Zhang, T. (September 07, 2003). Generating Panorama Photos.

[11] Szeliski, R. (December 10, 2006). Image Alignment and Stitching. Tech. rep. 
[12] Bergen, J. R., Anandan, P., Hanna, K. J., \& Hingorani, R. (1992). Hierarchical Model-Based Motion Estimation.

[13] Yanfang Li, Y. W. (2008). Automatic Image Stitching Using SIFT.

[14] Bay, H., Ess, A., Tuytelaars, T., \& Van Gool, L. (2008, Jun). Speeded-Up Robust Features (SURF). Russol Abdelfatah, H. O. (2013). Automatic Seamless of Image Stitching. International knowledge shating platform, 4, ISSN (Paper) 2222-1727.

[15] Ke, Y., \& Sukthankar, R. (2004). PCA-SIFT: A more distinctive representation for local image descriptors.
[16] Dalal, N., \& Triggs, B. (2005). Histograms of Oriented Gradients for Human Detection.

[17] Jiaxi Wang and Junzo Watada, Member, IEEE,

[18] D. Marr and E. Hildreth, - Theory of Edge Detection, Proc. of Royal Soc. London B, vol.

[19] Mrs. Hetal M. Patel, A. P. (November- 2012). Comprehensive Study and Review of Image Mosaicing Methods. 